\title{
Contextual Risk Analysis for Interview Design
}

\author{
Tira Cohene and Steve Easterbrook \\ Department of Computer Science, \\ University of Toronto, Toronto Canada \\ tcohene@dgp.toronto.edu,sme@cs.toronto.edu
}

\begin{abstract}
Interviews with stakeholders can be a useful method for identifying user needs and establishing requirements. However, interviews are also problematic. They are time consuming and may result in insufficient, irrelevant or invalid data. Our goal is to re-examine the methodology of interview design, to determine how various contextual factors affect the success of interviews in requirements engineering. We present a case study of a Web conferencing system used by a support group for spousal caregivers of people with dementia. Two sets of interviews were conducted to identify requirements for a new version of the system. Both sets of interviews had the same information elicitation goals, but each used different interview tactics. A comparison of the participants' responses to each format offers insights into the relationship between the interview context and the relative success of each interview technique for eliciting the desired information. As a result of what we learned, we propose a framework to help analysts design interviews and chose tactics based on the context of the elicitation process. We call this the contextual risk analysis framework.
\end{abstract}

\section{Introduction}

In requirements engineering (RE), analysts gather data in order to learn about the users' needs, abilities, goals, and system requirements. Direct interviews are a common method of eliciting requirements $[1,16,31]$. However, interviews are notoriously problematic, and may yield insufficient, irrelevant, and/or erroneous data. The interview settings can create biases, communication problems, and social issues. Today's increasing range of system users and environments adds challenges such as dynamic and complex requirements.

The importance of context has been recognized in a number of requirements elicitation techniques, most notably, ethnography $[17,32,34]$ and contextual inquiry [4]. In this paper, context refers to the conditions that surround an element that determine how that element is interpreted. This includes the physical elements of the system and the environment. System context refers to any factors around an interactive system that contribute to the way the system is used. In addition to elements of the system and the environment, this can include the stakeholders' abilities and goals, as well as social factors. Socially-based systems involve especially challenging contexts. For example, health care systems often have intricate system contexts because they handle a substantial amount of personal data. This can lead to many unforeseen design challenges.

In this paper, we describe our work on the requirements elicitation process for a system with a particularly demanding social context. Caring For Others (CFO) is a web-based support tool for caregivers of people with dementia [3]. The system provides information, training, and access to videoconference support groups. Before we joined the CFO project, the original CFO researchers interviewed users in order to evaluate the system. Unfortunately, the resulting interview data was not sufficient for understanding the users' requirements. Various factors in the interview setting affected the validity of the responses, and meant that much of the data was irrelevant for our purposes.

We set out to design an interview format that elicits more productive responses. An effective interview is one that can collect sufficient, relevant and appropriate data, so that requirements can be produced [27]. We also wanted to minimize the risk that problems in the interview setting would lead to erroneous data.

We begin the paper with an introduction to the CFO system and the evaluation techniques conducted for it. We then discuss interviews, the common problems associated with them, and some proposed solutions in the field. We introduce a risk analysis approach to interview design, and show how we applied it to the CFO project. We conducted a qualitative analysis on the results of our interviews and compare the results to the original evaluation. We outline the significant differences, and show how the second method helped elicit new requirements for the $\mathrm{CFO}$ system. Based on these findings we propose a framework to help analysts choose interview tactics and design their interview.

\section{The CFO Project}

Caring For Others (CFO) is a support tool for seniors who care for their spouses with dementia. The tool was developed in 2003 at the Baycrest Centre for Geriatric Care, headed by Dr. Elsa Marziali, Schipper Chair in Gerontological Social Work. The research team, with 
backgrounds in gerontology and social work, administered the initial design and development for CFO. All research discussed in this paper was conducted with guidance from the research team, ethical approval from Baycrest, and informed consent from participants.

CFO is a web-based portal for access to information, training, and videoconference support groups. Once logged on, participants can access private videoconference groups, resources on dementia and caregiving, or chat and email with other group members. Figure 1 shows a screen capture of the CFO home page.

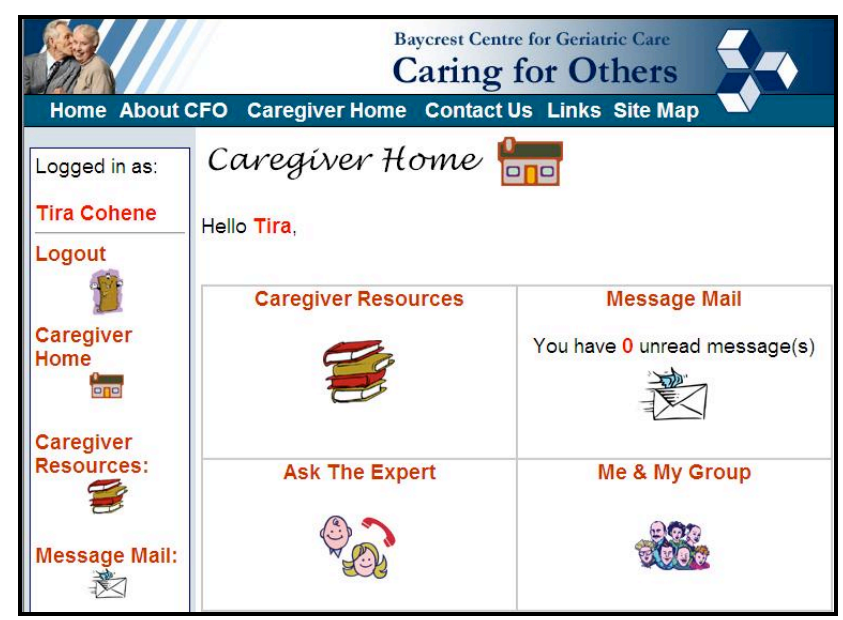

Figure 1: The CFO Homepage

Support of this nature has a wide range of benefits. The system helps participants communicate with other caregivers in similar circumstances. It also facilitates communication with professional health care providers, family, and friends. The enhanced communication can help reduce the stress and burden of caregiving. The CFO system requires fewer resources than face-to-face support groups. In addition, Internet support can be made available to people regardless of their location and without having to leave the person with dementia.

This project has various psychosocial requirements. The researchers wanted the system:

- To meet participants' expectations of a support group;

- To help participants cope with feelings;

- To help participants learn to manage caregiving tasks;

- To familiarize participants with computers.

We next discuss how the CFO researchers evaluated these requirements and learned about the $\mathrm{CFO}$ usage.

\subsection{The CFO Evaluations and Assessment}

The original CFO researchers conducted an interview to study the effects of using the system after six months of website access. Participants logged on to the system at least once a week and at most on a daily basis. Twentyeight users participated in these original interviews. The researchers had three particular goals for the interview:
1. To determine whether the user's requirements had been met;

2. To evaluate the benefits of using the system;

3. To gather requirements for system enhancements.

The interview included open-ended and closed-ended questions that were designed to meet these goals.

The majority of the interview questions were direct questions that asked users to relate personal experiences. Sample questions include:

- Did you find the website easy to use?

- What did you like most about the website?

- What did you like least about the website?

- Did connecting with other caregivers via the website meet your expectations of how a support group could

help you cope with the stress of caregiving?

Interview topics included how participants use the system, how the system affects participants, and how participants feel about the system. Results of this interview indicated that the majority of participants found the videoconference support group to be extremely valuable.

However, the data gave little insight into how the system could be improved. For example, when asked if they found the website easy to use, $100 \%$ of the respondents said 'yes'. When asked what they liked least about the website, participants complained about a specific technical problem that was eventually resolved. Many said that there was "nothing to complain about at all". In some cases respondents left questions unanswered because they couldn't recall specific experiences.

We wanted to create a more effective interview process with sufficient, relevant data for understanding the user needs and system requirements. In the next section, we discuss interviews and the common methodologies proposed in the RE and system design fields.

\section{Interviews}

Interviews for RE generally involve asking stakeholders questions about the current system that they use or the system to be developed. The most common type of interview is face-to-face. Questionnaires, focus groups, telephone, and Internet interviews are common variants. The interviews can be structured, semistructured, or unstructured. Interviews have the potential to elicit a large amount of information. They are valuable for gaining a global understanding of the stakeholders' behaviours and environments [29,37], including tasks, system interactions, and personal feelings. They also help to effectively elicit habitual practices and social factors [1].

In structured interviews, interviewers ask a set of predefined questions. In contrast, semi-structured interviews can be delivered in an exploratory and dynamic manner. When an interviewer varies his or her techniques this is known as employing tactics [12]. For example, an interviewer can change question formats or include 
narratives, scenarios, or role-playing. Other tactics common in social sciences include free listening, card sorts, triad tests and paired comparisons [28]. Such tactics provide analysts and participants with more means of communicating socio-technical issues [24]. In unstructured interviews, there is no pre-defined agenda, and interviewers may explore a range of issues with stakeholders.

Throughout this paper, we use the term interview to refer to elicitation processes based primarily on asking questions, even when other elicitation tactics are included in the process.

\subsection{Problems with Interviews}

Several factors in the interview setting are difficult to control and lead to problematic data. For example, many interview formats assume that the participant has explicit knowledge of their needs in a system. This is especially difficult when the system does not exist or when the system depends greatly on social and political issues [1].

Participants are subject to the limitations of their own memory and communication abilities. Studies in cognitive science show that memories can be lost, distorted or blocked [21,30]. Tacit knowledge may be hard to articulate, leading to the say-do problem, where people are unable to describe what they do [15]. For example, the act of tying shoelaces is easier than verbally describing how to tie shoelaces. Other issues arise from communication problems such as domain terminology, shared meaning, ambiguity, or biases [6,15,31]. For example, an analyst may use language in an interview that is unclear or misleading to the stakeholder, and vice versa.

Social factors such as status, gender, and environment may also lead to problematic data [15]. For example, a system may involve personal or private contexts of use, and participants may not feel comfortable relating personal feelings or experiences.

Theories from cognitive science can also explain how participants' responses may be inaccurate. Cognitive biases refer to distortions in the way people see reality [14]. For example, in-group bias occurs when people give preferential treatment to members of their groups [35]. This preferential treatment may lead to biased responses from participants. Another example is the framing effect [36]. When two decision questions are framed in alternative ways, they can lead to systematically different choices by respondents. In some cases this occurs because people tend to choose risk aversion options for gains and risk seeking options for losses. As a result of the framing effect, people violate principles of rational decision making, and will not always choose the rationally optimal choice.

To cope with the problems that arise from these communication factors, cognitive factors, and social factors, researchers offer various guidelines, techniques, and methodologies.

\subsection{Previous Work: Enhancing Elicitation}

What can analysts do in order to enhance the interview design and the elicitation process? Interviewers often share their expertise by offering basic guidelines. In order to conduct an effective interview, interviewers should be open-minded, unbiased, and ask meaningful questions [31]. Other sources suggest strategies for effective communication such as creating a point of reference and shared meaning [26], or offer a toolbox with sample questions and techniques [13].

Strategies and heuristics are often acquired through experience $[16,20]$. In a recent study, Hickey and Davis examined how experts select elicitation techniques [16]. According to this study, experts select techniques as a result of both the information they are trying to find and the conditions necessary to conduct the technique. However, Hickey and Davis conclude that more research is needed to understand the relationship between different system contexts and technique selection. They suggest that further research can consider which techniques may be optimal under a set of conditions.

Many of the problems with interviews are related to language and communication. Some tactics avoid this problem by shifting the focus from oral responses to physical activities. These methodologies have helped analysts understand users' needs as a result of what they do, rather than what they say [5]. Techniques such as ethnography [17,32,34], contextual inquiry [4], and usability studies look at activities as they occur in their natural environment. For example, in the protocol analysis technique, analysts observe users as they conduct a task and describe aloud what they are thinking.

Contextual inquiry (CI) is an elicitation process that develops an explicit understanding of users' needs, desires, and work models. In CI, interviewers elicit customer needs in their environment or workplace. Beyer and Holtzblatt [4] identify several goals that distinguish this approach from other interviewing strategies. In CI, analysts make observations within the customer's context, discuss the observations as they happen, and determine the implications for the design. They should also focus the interview towards meaningful, unbiased data.

Beyer and Holtzblatt [4] also provide additional guidelines that help analysts decide how to set up the interview. For example, an interview can be an effective elicitation technique in normal workplace scenarios. However, when the work requires a large amount of focus from the participant it's better to videotape the event and view it in more detail later in a retrospective interview session. These guidelines provide more insight into the relationship between the system context and the technique selection. They suggest that elicitation techniques should be selected based on activity factors such as the duration, the frequency, and the attention required.

Other research provides a further analysis of the interview design itself. In [38], Wood suggests selecting tactics based on the type of information that the analyst is 
trying to find. For example, different types of questions are appropriate for (I) identifying objects, (II) discovering relationships between objects, and (III) learning about the customer's process knowledge. As an example, in order to identify a work place object, Wood suggests eight different question formats (table 1).

\begin{tabular}{|l|l|}
\hline Question Tactic & Example \\
\hline Task-Related & Could you discuss the steps in... \\
\hline Guided & Could you show me... \\
\hline Typical & $\begin{array}{l}\text { Could you tell me about a } \\
\text { typical... }\end{array}$ \\
\hline Example & Can you show me an example of... \\
\hline Personal Experience & $\begin{array}{l}\text { Could you tell me about some of } \\
\text { your experiences? }\end{array}$ \\
\hline Direct Language & What do you call... \\
\hline Hypothetical-Interaction & How would you describe... \\
\hline Use & $\begin{array}{l}\text { What purpose does this feature } \\
\text { serve? }\end{array}$ \\
\hline
\end{tabular}

Table 1: Object Identification Questions (Adapted from [38])

In addition to these question formation tactics, Wood suggests that scenario development is also important for eliciting detailed descriptions [38]. Scenarios [7,8] are commonly used in user-centered design, most often to describe activities or conceptual models. This is mostly done within the design and development groups, however researchers have also used them in participatory activities to generate requirements [8].

Personas [9] are another form of narrative that are engaging and help provide information about the users. Unlike scenarios, which focus on activities, personas focus on fictional characters. Analysts often use data from interviews in order to generate personas. Yet little research examines the use of personas as a tactic to elicit data during interviews.

In many other domains, researchers acknowledge that some tactics are more effective than others in certain contexts. Social scientists [12] discuss how techniques vary with the group being interviewed. For example, interviewing children [11] requires a different approach than interviewing widows [23]. Unfortunately, the researchers do not provide specific details on these different approaches.

The literature shows how analysts may enhance the elicitation process by altering their tactics. This includes employing techniques from $\mathrm{CI}$, altering question formation, or using scenarios and personas. Is there a way to select elicitation tactics such as these while minimizing the problems within the interview process? In order to investigate this question we conducted a case study with the CFO participants. First we explore the special nature of the context of the CFO system. Based on this, we designed an interview protocol that employs specific tactics. The process that led us to the design of the interview is discussed next.

\section{Contextual Risk Analysis for Interviews}

There are many contextual factors in the CFO system that can affect the needs and requirements of the system. The same factors can also affect the information gathering process. Our first goal is to understand how the CFO context might affect the interview process. Therefore, we begin with a detailed description of the system context.

\subsection{System Context of CFO}

The context of the CFO system is unlike many of the traditional environments that are researched in system design. The research environments in this and related domains have "traditionally focused on tools and techniques to be used on adult users involved in large scale, office based systems development. The research has rarely focused on educational, entertainment, innovative and personal applications" [24]. The CFO system provides an interesting research environment because it allows us to study a wide range of contexts. It is becoming increasingly important to study conditions such as users with special needs or social biases because of the growing amount of conditions in new systems.

The primary stakeholders of CFO are individuals who care for their spouses with dementia. Dementia is a progressive brain disease causing a decline in cognitive abilities. The most common form of dementia is Alzheimer's disease. Common symptoms of dementia $[2,18,19]$ are listed in table 2 . For further information on dementia see [22].

\begin{tabular}{|ll|}
\hline Cognitive Symptoms: & Behavioural Symptoms: \\
- Gradual loss of memory & - Personality changes \\
- Problems with reasoning & - Agitation \\
or judgment & - Anxiety \\
- Disorientation & - Delusions (believing in a \\
- Difficulty in learning & reality that does not \\
- Loss of language skills & exist) \\
- Decline in the ability to & - Hallucinations (seeing \\
perform routine tasks & things that do not exist) \\
\hline
\end{tabular}

Table 2: Symptoms of Dementia

The loss of thinking skills, including memory loss, can disrupt an individual's ability to perform everyday tasks. As these capabilities degenerate, so does the individual's independence. The person will rely on others to help compensate for their diminishing capabilities.

The caregiver, who is often a family member such as an elderly spouse, becomes very much affected by the responsibilities of caregiving. Studies show that caregivers often neglect their own health and wellbeing as a result of the stress of caregiving [10]. Many older individuals are socially isolated due to a shrinking network of family and friends [25]. Many caregivers also 
cannot leave their ill spouses unattended [3]. Support networks are invaluable for providing communication, compassion, advice, and consolation. The nature of the support is an essential element of the CFO context.

The current system design is also an important factor of the system context. The majority of the spousal caregivers are over the age of sixty, and inexperienced computer users. As a result, the system accounts for various factors such as failing eyesight, decreasing muscle coordination, difficulty remembering recently learned concepts, and an overall decrease in the rate of learning. The system also accounts for a range of user abilities. These factors are addressed in the CFO project through hardware enhancements, interface design choices, and specialized training sessions. Figure 2 shows one example of how the system design accounts for failing eyesight.

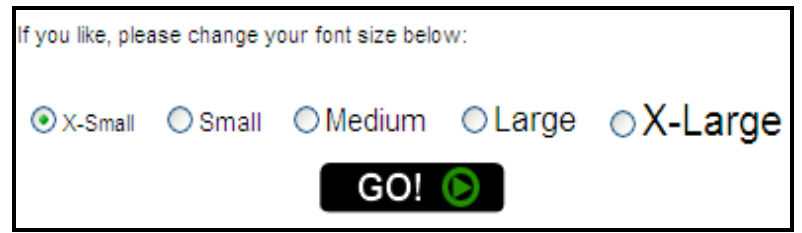

Figure 2: CFO and the Needs of Older Adults

After reviewing the results of the first interview, we became aware of several risks in the system context. These risks are factors that may impede both the design of the system and the design of our elicitation techniques. They include:

- Personal and Social Factors: The interview will need to minimize pressures and biases faced by participants

- Information and Communication: The interview language will need to support open and effective communication with elderly caregivers

- Cognitive Factors: The interview will need to be sensitive to issues such as cognitive biases, as well as age-related issues such as memory, computer use, and computer experience

The following section describes how we designed our interview process after taking these contextual risk factors into consideration.

\subsection{Study Design}

In this exploratory case study we compare two interview processes designed with different methodologies. The researchers designed the first interview based on the information that they were trying to find, i.e. the three interview goals discussed in section 2.1. The second interview was designed in order to both (a) meet the interview goals (section 2.1) and (b) reduce the risks involved in the elicitation process. Our hypothesis is that a contextual risk analysis for the interview design will better satisfy the goals of our interview.
The contextual risk analysis for our interview design included:

1. Investigating the $\mathrm{CFO}$ system context

2. Identifying the $\mathrm{CFO}$ contextual risks

3. Identifying the interview goals

4. Researching various elicitation techniques

5. Selecting techniques to reduce contextual risks

The variables in this study are the interview tactics such as the question format, the question framing, or the language. The control variable is the type of information that the question is intending to elicit. We intend to compare questions using different tactics that have similar elicitation intentions. That is, we compare how different questions or tactics that intend to elicit similar data may lead to different responses. In order to measure the differences among different question tactics we will look at the number of themes and categories identified in the data, the range of responses, the depth and quality of the responses, as well as the amount of critical feedback.

We interviewed five CFO users who did not participate in the first follow up interview. It was important that these participants had not taken part in the first interview so that the two sets of responses could be independent. The five participants were exposed to the same version of the system under similar circumstances as the twenty-two participants in the first interview. Although this group was smaller than that in the first interview, the groups both displayed similar variances in conditions such as age, gender, and education levels. Since circumstances prevented us from having the same interviewer conduct the two interviews, we tried to minimize the effects of these differences. The second interviewer met with the research team, studied audio transcripts from the first interview, and attempted to replicate as many factors as possible within the interviewing conditions.

The second interview included various question formats, scenarios, and a protocol analysis. An example of a question format includes hypothetical experience questions (adapted from [38]). Hypothetical experience questions involve personas or hypothetical characters. Each hypothetical experience describes a person in a specific situation who is considering using the CFO system. The participants were asked to give their advice and describe how joining CFO might affect each person.

The interview also included a protocol analysis. We asked participants to imagine that the interviewer was a new member of CFO and had never used the website before. We then asked them to teach the interviewer how to use the system. In this tactic the think-aloud became a teach-aloud where participants described what they were doing in order to help a new user understand the system.

Following the protocol analysis were case focused questions and guided questions. These questions were related to the participants' experiences using CFO, however they did not require explicit personal information about the participant. These questions were designed to 
allow users to describe their needs regarding system elements where personal factors did not play a role.

We conducted a qualitative analysis of the interview data and found various themes and categories, described by qualifiers and examples (for information on qualitative analysis for social sciences see [33]). The results of the qualitative analysis showed that the questions in this interview stimulated thoughtful responses regarding contexts of use. They helped participants reflect on their own experiences in a comfortable and natural context.

\section{Results}

When comparing the qualitative analysis results of the first and second interviews, we found many common categories and themes. However, in several cases of the second interview the responses were significantly different. The second interview also identified up to twice as many themes for certain questions. Throughout this section we discuss these differences with respect to the risks we uncovered for this interview design.

\subsection{Risk 1: Personal and Social Factors}

We employed several tactics to minimize any personal or social factors in the interview context. The interview included a protocol analysis, a tactic that looks at what users do as opposed to what they say. In the context of CFO, where participants are generally new computer users, it was important to maintain a natural atmosphere. In this protocol analysis participants were asked to 'teach' the interviewer how to use the system. This takes the pressure away from the test-like nature that may be associated with most think-aloud techniques.

This protocol analysis uncovered new usability issues, mostly related to computer experience and age. In the first interview, $100 \%$ replied that the website is easy to use. However, in the second interview during the protocol analysis the majority of the participants $(80 \%)$ exhibited minor difficulties when using the site. For example, participants clicked on text instead of links or icons. Other common issues involved navigation and typing errors.

Many of the questions in the first interview were personal in nature. They asked how participants cope with upsetting feelings and deal with the loss of their spouse. In the first interview many of the responses are distributed to extremes. Participants described their experiences as 'completely fine' or 'completely terrible'. In the second interview, topics such as coping and loss are discussed in terms of a hypothetical person. Responses to these hypothetical questions appear more distributed and sincere. They described how the system affects someone as opposed to how it affects them. They described general overall feelings about the system. In several cases participants freely related personal experiences that supported their responses.

\begin{tabular}{|c|c|}
\hline $\begin{array}{l}\text { Interview 1: Personal } \\
\text { Experience Question }\end{array}$ & $\begin{array}{l}\text { Interview 2: Hypothetical } \\
\text { Experience Question }\end{array}$ \\
\hline $\begin{array}{l}\text { From your experience in } \\
\text { this project what advice } \\
\text { would you give to the } \\
\text { researchers about the } \\
\text { design and ease of use } \\
\text { of the website? }\end{array}$ & $\begin{array}{l}\text { Scenario: Norma doesn't like } \\
\text { technology. She doesn't own } \\
\text { an answering machine and } \\
\text { she doesn't like using the } \\
\text { VCR. Recently, Norma's been } \\
\text { considering joining the } \\
\text { videoconference group. } \\
\text { Questions: } \\
\text { a) Would you recommend the } \\
\text { videoconference group to } \\
\text { her? Why? } \\
\text { b) What kinds of benefits } \\
\text { would you tell Norma that she } \\
\text { can expect from using the } \\
\text { website? }\end{array}$ \\
\hline \multicolumn{2}{|l|}{ Sample Responses: } \\
\hline $\begin{array}{l}\text { "I think it was long } \\
\text { enough." } \\
\text { "It's a very useful tool." } \\
\text { "I have no } \\
\text { suggestions...I think } \\
\text { they did a great job with } \\
\text { that website. They made } \\
\text { it simple for people like } \\
\text { me that could learn it } \\
\text { easy and it worked." } \\
\text { "To work out some of the } \\
\text { computer glitches." } \\
\text { "It seemed relatively } \\
\text { easy to use once the } \\
\text { bugs were ironed out." } \\
\text { "It's a good project, if } \\
\text { the computer had worked } \\
\text { properly." }\end{array}$ & $\begin{array}{l}\text { a) "Yes. It's natural for her to } \\
\text { resist it at first." } \\
\text { "It's possible for someone } \\
\text { who's never used the } \\
\text { computer to use the website." } \\
\text { "With training and experience } \\
\text { she'll be more comfortable." } \\
\text { b) "Helps in obtaining } \\
\text { information." } \\
\text { "A useful tool for talking to } \\
\text { people, forming relationships, } \\
\text { getting their opinions and } \\
\text { support." } \\
\text { "Become more confident with } \\
\text { computers." }\end{array}$ \\
\hline
\end{tabular}

Table 3: Sample Responses to Question III

In the first interview, one question asked about the website design and usability (table 3 ). In the second interview we framed the question in terms of a fictional persona. The hypothetical experience question also aims to elicit how the website is useful and usable, in terms of someone who is afraid of technology. The responses to the question in the first interview were less informative for our interview goals than those in the second. In the first interview the majority of the responses fell into two categories. Participants said there were no problems at all, or they referred to specific 'bugs' or 'glitches' that were resolved right away. In the second interview, the responses address more themes, particularly social issues. They refer to user attitude, computer training, and access to resources. These responses offer additional insight on the value of particular themes and they inspire psychosocial requirements for system enhancements. 


\subsection{Risk 2: Information and Communication}

The hypothetical experience questions helped to communicate and establish an initial reference point for the context of the entire system, something that is often missing in discussions and interviews [20]. In the first interview, one question asked what participants liked least about the website (table 4). The goal of the interviewers was to learn about some of the system drawbacks and problem areas. In the second interview, we altered the elicitation tactic and established a context for the participants. In order to determine the drawbacks, we compared the system to in-person support groups.

\begin{tabular}{|c|c|}
\hline $\begin{array}{l}\text { Interview 1: Personal } \\
\text { Experience Question }\end{array}$ & $\begin{array}{l}\text { Interview 2: Hypothetical } \\
\text { Experience Question }\end{array}$ \\
\hline $\begin{array}{l}\text { What did you like least } \\
\text { about the website? }\end{array}$ & $\begin{array}{l}\text { Scenario: John currently } \\
\text { participates in face-to-face } \\
\text { group support for caretakers. } \\
\text { He can either continue to go to } \\
\text { his current support group, or } \\
\text { try out the videoconference } \\
\text { group. } \\
\text { Question: What would be the } \\
\text { benefit of staying in his current } \\
\text { support group? }\end{array}$ \\
\hline \multicolumn{2}{|l|}{ Sample Responses: } \\
\hline $\begin{array}{l}\text { "Well, we had to make } \\
\text { appointments to be } \\
\text { able to connect with } \\
\text { each other and stick to } \\
\text { it." } \\
\text { "Connection problems } \\
\text { with the meeting." } \\
\text { "It was all helpful." } \\
\text { "I can't remember..." }\end{array}$ & $\begin{array}{l}\text { "In the early stages of } \\
\text { caregiving, face-to-face contact } \\
\text { is more advantageous - body } \\
\text { language, facial expressions, } \\
\text { eyes..." } \\
\text { "Sometimes it's hard to put } \\
\text { caregiving on pause to use the } \\
\text { computer." } \\
\text { "Getting out of the house is } \\
\text { good." } \\
\text { "The technical issues might get } \\
\text { in the way of getting support." }\end{array}$ \\
\hline
\end{tabular}

Table 4: Sample Responses

Without context, participants often focused their answers on specific tasks or instances of use, such as the videoconference application. In the second interviews, the responses pertained to the entire system and how it's used. Table 4, listed below, shows some of the typical responses to these questions.

Establishing reference points also helps to define ambiguous words and create shared meaning. A question in the first interview included the term improving the system (table 5). To many participants improving meant adding features. In the second interview, the question is framed specifically in reference to system usability. As a result of this, participants addressed a range of usability issues in addition to new features.

Some questions may assume that users have explicit knowledge of their needs in a system. For example, the question in the first interview, "How might they [the researchers] improve the design and ease of the website?" will only elicit meaningful information if the participants can foresee potential changes in the system (table 5). This can be challenging, especially for novice computer users. In the second interview, the subject is approached in terms of an actual experience, not a comparison of potential elements. Participants can freely discuss what they know as opposed to novel concepts.

\begin{tabular}{|c|c|}
\hline $\begin{array}{l}\text { Interview 1: } \\
\text { Contrast Question }\end{array}$ & $\begin{array}{l}\text { Interview 2: Hypothetical } \\
\text { Experience Question }\end{array}$ \\
\hline $\begin{array}{l}\text { How might they improve } \\
\text { the design and ease of } \\
\text { use of the website? }\end{array}$ & $\begin{array}{l}\text { Scenario (from Table 3). } \\
\text { Question: Do you think } \\
\text { Norma will ever get } \\
\text { frustrated or confused when } \\
\text { using the website? If yes, } \\
\text { what specific parts of the } \\
\text { website will make her feel that } \\
\text { way? }\end{array}$ \\
\hline \multicolumn{2}{|l|}{ Sample Responses: } \\
\hline $\begin{array}{l}\text { "I don't think there's } \\
\text { anything that should be } \\
\text { different." } \\
\text { "People should meet in } \\
\text { person before the start of } \\
\text { the group online." } \\
\text { "I would definitely have } \\
\text { a forum. The email is } \\
\text { good but I don't think } \\
\text { you get as much out of it } \\
\text { as a forum... where you } \\
\text { could post your } \\
\text { questions and anybody } \\
\text { could answer them." }\end{array}$ & $\begin{array}{l}\text { "The kinds of things that are } \\
\text { confusing are general } \\
\text { computer use issues - mouse, } \\
\text { network connections, } \\
\text { hardware, etc., not issues } \\
\text { specific to the website." } \\
\text { "Accessing it and using it } \\
\text { could be simpler and quicker. } \\
\text { In the conference you can't } \\
\text { speak all at once, it's a } \\
\text { turntable." } \\
\text { "After time it becomes habit." }\end{array}$ \\
\hline
\end{tabular}

Table 5: Sample Responses to Question IV

A final question in the interview asked users to reflect on their own experience. Unlike the other hypothetical questions, this question is personal. We asked, "If you could see yourself when you first started caring for your spouse, would you have any advice to give yourself?" This question is a natural language question [38], exploiting the participants' inherent language and communication abilities. In support groups, participants are familiar with offering advice and understanding. It also elicits participant needs from a previous time without asking them to recall specific events or feelings.

\subsection{Risk 3: Cognitive Factors}

The hypothetical nature of the scenario can help reduce pressures that the participants may feel to please the interviewers. Participants may not want to risk offending the researchers by relating negative comments. In the first interview, participants were asked, "Did the participation in the group help you to manage tasks of caregiving more effectively". Seventy percent of the participants responded positively ( $10 \%$ responded 'perhaps' and $20 \%$ responded 'no'). In the second interview, participants were given the following scenario: 
Ellen cares for her husband Bill on a full time basis. Recently, caring for her husband has been taking up much more of her time than it used to. It's become very challenging to manage all of the caregiving tasks such as organizing meals, medication, household chores, and the various appointments.

They were then asked, "Do you think that joining the video conference group will make a difference in Ellen's ability to manage tasks?" In contrast to the first interview, none of the participants responded positively $(60 \%$ responded 'perhaps' and 40\% responded 'no').

Two other closed ended questions were asked in each interview to compare the two tactics. However there were no significant differences between the two interviews. An interesting note is that the majority of the responses for the other two questions in both interviews were positive. When the participants were responding positively, they may not have felt pressure to bias the answers. However, when the participants wanted to respond negatively, there may have been pressure to lie. This may be consistent with the cognitive biases discussed in section 3.1.

By focusing on a hypothetical person and that person's situation, the participants are not required to relate any personal experiences. Thus participants are not relying on their own memory. In the first interview, participants sometimes could not answer questions because they could not recall specific circumstances. In the second interview, we were able to elicit a larger amount of feedback.

\section{Contextual Risk Analysis Framework}

This case study investigates the effect of scenarios describing the system context, question types, and techniques for framing users' answers on the type of requirements information acquired. For example, we found that hypothetical experience questions are an effective way to avoid many of the risks associated with information and communication factors. As a result of the additional themes in the data, we found previously hidden requirements such as the need to support a CFO community, support communication conventions in the videoconferencing feature, support continuous and dynamic training and resources, and resolve various usability issues. These requirements will be addressed in upcoming $\mathrm{CFO}$ versions that will include enhancements to meet these requirements.

In previous work on elicitation techniques, researchers suggested creating a tool that uses expert knowledge to map contexts into the set of appropriate elicitation techniques [16]. Unlike much of previous research on elicitation technique selection, the driving force behind tactic selection here focuses on the risks in the system context. We can begin creating this tool by mapping the contextual risks against our tested elicitation techniques from the second interview. In table 6 we list our findings related to the contextual risks for the CFO system.

\begin{tabular}{|c|c|c|}
\hline $\begin{array}{l}\text { Contextual } \\
\text { Risks }\end{array}$ & Tactics Used & Affects \\
\hline \multirow[t]{2}{*}{$\begin{array}{l}\text { Personal \& } \\
\text { Social Factors }\end{array}$} & $\begin{array}{l}\text { Protocol } \\
\text { Analysis }\end{array}$ & $\begin{array}{l}\text { Overcome say/do } \\
\text { problem }\end{array}$ \\
\hline & $\begin{array}{l}\text { Hypothetical } \\
\text { Experience } \\
\text { Question }\end{array}$ & $\begin{array}{l}\text { Not required to relate } \\
\text { personal experiences } \\
\text { Less pressure to } \\
\text { please interviewer }\end{array}$ \\
\hline \multirow[t]{2}{*}{$\begin{array}{l}\text { Information \& } \\
\text { Communication } \\
\text { Factors }\end{array}$} & $\begin{array}{l}\text { Hypothetical } \\
\text { Experience } \\
\text { Question }\end{array}$ & Establishes context \\
\hline & $\begin{array}{l}\text { Natural } \\
\text { Language } \\
\text { Question }\end{array}$ & $\begin{array}{l}\text { Exploits } \\
\text { communication } \\
\text { abilities }\end{array}$ \\
\hline $\begin{array}{l}\text { Cognitive } \\
\text { Factors }\end{array}$ & $\begin{array}{l}\text { Hypothetical } \\
\text { Experience } \\
\text { Question }\end{array}$ & $\begin{array}{l}\text { Not required to recall } \\
\text { specific events } \\
\text { Can manipulate } \\
\text { question framing }\end{array}$ \\
\hline
\end{tabular}

\section{Table 6: Relationship Table for Contextual Risk Analysis Framework}

The relationship table represents a seed for a framework that can grow to account for other tactics and contextual risks as more research is conducted. We also consider the drawbacks or tradeoffs of using specific tactics. For example, although hypothetical experience questions put the participant out of the focus, they may lead to alternative framing effects or biases. They may also elicit generalized responses and fewer personal experiences. These generalizations may not cover unusual use cases. This can be problematic if analysts wish to elicit every potential use case, or if they want to report the actual effects of the system. This is less problematic if the goal of the interviews is to gather general information about the current system, the users, and the contexts of use. We also found that participants often generalize based on their personal feelings and experiences.

Another tradeoff involves the effort and duration of conducting different tactics. Experts often select elicitation techniques based on the necessary conditions because the conditions can involve limited resources [16]. As a result of our research we've found that this can be problematic for several reasons. The technique that meets the necessary conditions may not be the most effective elicitation technique. In addition, many techniques require surprisingly different resources. For example, we found that the hypothetical experience questions take a little longer to create and pose to the participant, yet the responses tended to be significantly shorter because they involved fewer personal accounts.

We are aware that this process involves a thorough understanding of both the system context and the tradeoffs 
involved in the selection of tactics. This research also explores a specific context and a small sample size. However, we believe that it shows how we need to have a better understanding about the relationship between contextual framing and requirements elicitation design.

\section{Future Work}

In future work, we hope to explore other interview tactics and system contexts. Methodologies such as phenomenology, grounded theory, or ethnomethodology [15] may offer significant insight. In addition to these interviewing tactics, we hope to explore elicitation tactics such as team building and role-playing.

System contexts with atypical stakeholders and environments require further study as well. For example, when interviewing in a critical safety context, how will different question formats affect the quality of the data? Further examples include contexts with dense amounts of data, contexts with culturally significant issues, or contexts with individuals with special needs.

We hope to conduct further research, such as a survey of contextual risk analyses, in order to verify and develop our hypothesis. Perhaps additional analyses other than those based on risks will be beneficial for the elicitation process in different ways. In addition, we hope to learn more about how the contextual risk analysis will affect the rest of the RE process as well as the system design.

In previous work there is little guidance offered on elicitation technique selection in relationship to the system context. With this framework, analysts in challenging design environments can consult the framework to determine which techniques are optimal under a set of conditions. For example, in the future, the framework may include a more thorough description of tactics used to elicit data from users who have difficulty communicating their needs. An analyst who is designing a novel application for users with Autism can consult the framework for useful elicitation tactics.

\section{Conclusions}

In this paper we present a case study that shows how contextual risks can affect the elicitation process. We identified three areas of contextual risk that can lead to omissions or errors in data collected through interviews: personal and social factors; information \& communication factors; and cognitive factors. When we took these contextual factors into account in our interview design, we found that we elicited more sufficient, relevant and valid data. By addressing the first risk, we isolated relevant issues in the protocol analysis. We also found that the responses were more informative for our elicitation goals. By addressing the second risk, we found that the participants focused on valuable elements such as the entire system and how it is used. The scenarios resulted in a larger amount of feedback. In response to the third risk, the hypothetical question tactic that may have helped avoid cognitive biases.

The research in this paper explores interview design, a process that is often neglected, or explained by heuristics and expertise. The tendency is to select elicitation techniques based on the kind of information one is trying to find, or the situational characteristics. Yet there is little guidance on how different techniques and situational characteristics affect the interview data. This paper has contributed some extensions to the existing methodology of requirements elicitation [4, 13] and tentatively demonstrated how different techniques elicit different types of information.

In response to our findings we proposed a framework for designing interviews that is based on contextual risks. The contextual risk analysis framework offers insight into this relationship and acts as a tool for future analysts to select optimal tactics. Historically, research design decisions have been made based on personal judgment in an opportunistic manner. Investigations such as these in this case study can help analysts make more informed decisions in their interview design and lead to enhanced data to support the RE and design process.

\section{Acknowledgments}

We thank the research participants, colleagues at the Baycrest Centre for Geriatric Care and the University of Toronto, and all those who provided valuable feedback and comments.

\section{References}

[1] Alvarez, R., and Urla, J. "Tell Me a Good Story: Using Narrative Analysis to Examine Information Requirements Interviews During an ERP Implementation," ACM SIGMIS Database, 33, 1 2002, pp. 38-52

[2] The Alzheimer's Association. "About Alzheimer's". 2004. http://www.alz.org

[3] The Baycrest Centre for Geriatric Care. "The Baycrest Bulletin",2,1. August 2003. http://www.baycrest.org/ reports/bulletin_aug2003.pdf

[4] Beyer, H. and Holtzblatt, K. Contextual Design: Defining Customer-Centered Systems. Morgan Kaufmann Publishers, San Francisco, CA, 1998.

[5] Broadbent, S., and Cara, F. "A Narrative Approach to User Requirements for Web Design. Proc. Interactions, ACM Press. 7, 6, 2000, pp. $31-35$.

[6] Byrd, T.A., Cossick, K.L., Zmud, R.W., "A Synthesis of Research on Requirements Analysis and Knowledge Acquisition Techniques", MIS Quarterly, 16, 1, 1992, pp.117-138.

[7] Carroll, J. (Ed.) Scenario-based design. Wiley. 1995.

[8] Chin, G., Rosson, M.B., \& Carroll, J.M. "Participatory Analysis: Shared Development of Requirements from Scenarios", in S. Pemberton (Ed.), Proceedings of CHI'97:Human Factors in Computing Systems. ACM Press/Addison-Wesley, New York, 1997, pp. 162-169. 
[9] Cooper, A. The inmates are running the asylum. Macmillan. Indianapolis. 1999.

[10] Czaja S. J., Rubert M. P. "Telecommunications Technology as an Aid to Family Caregivers of Persons with Dementia. Psychosomatic Medicine, 64, 2002, pp. 469-476

[11] Fine, G. A. and Sandstrom, K. Knowing Children: Participant Observation With Minors. Newbury Park, CA, SAGE, 1988

[12] Fontana, A., and Frey, J. H. "Interviewing: The Art of Science". In N. K. Denzin, \& Y. S. Lincoln (Eds.), Handbook of Qualitative Research, SAGE, Thousand Oaks, CA, 1994, pp. 361-376.

[13] Gause, D.C., and Weinberg, G.M. Exploring Requirements: Quality Before Design. Dorset House Publishing, NY, NY, 1989

[14] Gilovich, T., Griffin D. \& Kahneman, D. (Eds.). (2002). Heuristics and biases: The psychology of intuitive judgment. Cambridge, UK: Cambridge University Press.

[15] Goguen, J., \& Linde, C. "Techniques for Requirements Elicitation". Proc., First IEEE International Symposium on Requirements Engineering (RE'93), IEEE Computer Society Press, 1993, pp. 152-164.

[16] Hickey, A., and Davis, A., "Elicitation Technique Selection: How Do Experts Do It?" Proc., 11th IEEE International Requirements Engineering Conference (RE 2003), IEEE Computer Society Press, 2003, pp. 169-178.

[17] Hughes, J.A., Sommerville, I., \& Randall, D. "Designing with Ethnography: Making Work Visible". Interacting with Computers, 5, 2, 1993, pp. 239-253.

[18] Janssen-Cilag, "About Dementia". 2004. Retrieved on August 2, 2004 from www.dementia.com.

[19] Kasl-Godley, J., Gatz, M. "Psychosocial Interventions for Individuals with Dementia: An Integration of Theory, Therapy, and a Clinical Understanding of Dementia. Clinical Psychology Review 20,6, 2000, pp. 755-782.

[20] Kato, J., Komiya, S., Saeki, M., Ohnishi, A., Nagata, M., Yamamoto, S., and Horai, H. "A Model for Navigating Interview Processes in Requirements Elicitation" Eighth Asia-Pacific Software Engineering Conference (APSEC'01), 2001, pp.17-26.

[21] Kuberski, P., The Persistence of Memory: organism, myth, text. California UP, 1992.

[22] Lichtenberg, P.A. Handbook of Dementia : Psychological, Neurological, and Psychiatric Perspectives, John Wiley \& Sons, Canada, 2003.

[23] Lopata, H.Z. "Interviewing American Widows." In W. Shaffir, R. Stebbins, and A. Turowetx (Eds.), Fieldwork Experience: Qualitative Approaches to Social Research. St. Martin's, New York, 1980, pp. 68-81.
[24] Millard, N., Lynch, P., and Tracey, K., "Child's Play: Using Techniques Developed to Elicit Requirements From Children with Adults". Proc., Third International Conference on Requirements Engineering (RE'98), IEEE Computer Society Press, 1998, pp. 66-73

[25] Morris, M., Lundell, J., Dishman, E. "Catalyzing Social Interaction with Ubiquitous Computing: A Needs Assessment of Elders Coping with Cognitive Decline. Conference on Human Factors in Computing Systems. ACM Press, 2004, pp. $1151-1154$.

[26] Offen, R., "Requirements Elicitation and Modeling," 03/17/2004. Retrieved 08/02/2004 from http://www .comp.mq.edu.au/units/itec821/ITEC821_s104_week3. pdf

[27] Preece, J., Rogers, Y., Sharp, H. Interaction Design: Beyond Human-Computer Interaction. John Wiley \& Sons, Inc., New York, 2002.

[28] Romney, A. K., Weller, S. C., \& Batchelder, W. H. "Culture as Consensus: A Theory of Culture and Informant Accuracy". Am. Anthropo, 88. 1986. pp. 313 .. 338.

[29] Seidman, I., Interviewing as Qualitative Research: A Guide for Researchers in Education and the Social Sciences, $2^{\text {nd }}$ edition. Teachers College Press, New York, 1998.

[30] Schudson, M. 'Dynamics of Distortion in Collective Memory', in D.L. Schacter (ed), Memory Distortion, Harvard UP, 1995.

[31] Sommerville, I. Software Engineering, $7^{\text {th }}$ Edition. Pearson Education, 2004.

[32] Spradley, J.P. The Ethnographic Interview, Holt, Rinehart and Winston, New York, NY, 1979.

[33] Strauss, A. Qualitative Analysis For Social Scientists. Cambridge University Press, Cambridge, 1987.

[34] Suchman, L.A. Plans and Situated Actions: The Problem of Human-Machine Communication, Cambridge University Press, New York, NY, 1987.

[35] Tajfel, H. (1982). Social identity and intergroup relations. Cambridge, England: Cambridge University Press

[36] Tversky, A., and Kahneman, D. "Rational choice and the framing of decisions". Journal of Business 59, 4(2), 1986, pp. 251-278.

[37] Warren, C., "Qualitative Interviewing," in J. Gubrium and J. Holstein (eds.), Handbook of Interview Research: Context and Method, Sage, 2002, pp. 83-101.

[38] Wood, L.E. "Semi-Structured Interviewing for UserCentered Design”, Proc. Interactions, 4,2 1997, pp. 4861. 